\title{
Updating the VIVOPTIM Program for the Assessment of Cardiovascular Risk
}

\author{
Jacques Blacher' \\ Virginie Femery ${ }^{2}$ \\ On behalf of the VIVOPTIM \\ medical committee
}

'Diagnosis and Therapeutic Center, Hôtel-Dieu Hospital; Université de Paris; AP-HP, Paris, France; ${ }^{2}$ Department of Executive Management, VIVOPTIM Solutions, Paris, France
Correspondence: Jacques Blacher Université de Paris; Assistance PubliqueHôpitaux de Paris; Unité HTA, Prévention et Thérapeutique Cardiovasculaires, Centre de Diagnostic et de Thérapeutique, Hôpital Hôtel-Dieu, Place du Parvis Notre-Dame, Paris 75004 , France

Tel +33 | 42348966

$\mathrm{Fax}+33$ । 42348632

Email jacques.blacher@aphp.fr

\section{Plain Language Summary}

The VIVOPTIM program assesses individual cardiovascular risk and addresses modifiable risk factors with a view to reducing cardiovascular risk. Initially, the Framingham Risk Score was selected as the most appropriate method to determine cardiovascular risk. However, after analyzing the results of the pilot program, the steering committee concluded that the Framingham Risk Score was not the best suited. Therefore, before extending the VIVOPTIM program throughout France, a new cardiovascular risk assessment scale was developed, focusing on the six major cardiovascular risk factors (age, family history of early-onset cardiovascular disease, smoking, dyslipidemia, hypertension, and type 2 diabetes), plus seven associated cardiovascular risk factors with a 0.5 weighting (obesity, sedentary lifestyle, social deprivation, chronic inflammatory disease, kidney disease, chronic stress, and an unhealthy, non-Mediterranean-type diet).

In addition to the assessment of cardiovascular risk, participants also systematically receive information on excess risk, ie, risk factors that are preventable via measures of prevention: four major cardiovascular risk factors (smoking, dyslipidemia, hypertension, and type 2 diabetes), plus four associated cardiovascular risk factors with a 0.5 weighting (obesity, sedentary lifestyle, chronic stress, and an unhealthy, non-Mediterranean-type diet). This latter step, which is particularly important from an operational perspective, focusses on modifiable risk factors and provides constructive information and encouragement about the lifestyle improvements that each individual can implement to reduce their own cardiovascular risk.

Despite the major impact of cardiovascular morbimortality on healthcare statistics, ${ }^{1}$ and despite the widely recognized reversibility of many cardiovascular risk factors, neither individualized nor collective measures of prevention have to date been able to exert a significant impact on the incidence of modifiable risk factors within the population or on the promotion of healthy lifestyle attitudes in France, as in most countries. ${ }^{2}$ This observation led to the development of the VIVOPTIM program, which set out to assess individual cardiovascular risk and to assist in the management of cardiovascular risk factors using a personalized, interactive approach, novel information and communication technologies, and personalized coaching sessions conducted by specially trained professionals. ${ }^{3,4}$ This program is sponsored by the MGEN (a complementary health insurance in France). The short-term objective is to act on modifiable cardiovascular risk factors, namely diabetes, hypertension, smoking, and dyslipidemia, using essentially non-pharmacological tools, with the long-term aim of reducing cardiovascular risk and thus the incidence of cardiovascular morbimortality, and ultimately healthcare expenditure.

The pilot phase of the program was opened on a voluntary basis to all individuals (age, 30-70 years) insured by the MGEN in two regions of France (Bourgogne-Franche- 
Comté and Occitanie); 8883 volunteers were included between 15/11/2015 and 31/12/2017. Instead of a detailed evaluation of each individual's level of risk, our primary objective was to classify all participants into one of three cardiovascular risk groups (low, medium or high). Ascertaining the most appropriate cardiovascular risk scale has been a subject of extensive debate within the VIVOPTIM scientific committee. Both the Framingham ${ }^{5}$ and the SCORE $^{6}$ cardiovascular risk assessment scales are known to have several weaknesses, including their historical validity and their failure to consider several indisputable cardiovascular risk factors, such as a family history of early-onset cardiovascular disease, social vulnerability, obesity, sedentary lifestyle, chronic stress, chronic inflammatory disease, and renal failure, in particular.

SCORE had the benefit of a stronger geographic validity while the Framingham scale provided three clear advantages:

- An upper age limit of 74 years (versus 65 years in SCORE),

- Calculation of morbid cardiovascular events while SCORE only calculates cardiovascular deaths,

- More flexible use of HDL cholesterol and diabetes than in SCORE.

We therefore initially opted to use the Framingham risk score.

Box I Main Limitations to an Initial Cardiovascular Risk Assessment Based on the Framingham Equation

Few young participants in the highest cardiovascular risk group.

Young participants with several modifiable cardiovascular risk factors often ranked at low or moderate risk.

No specific rules for evaluation of risk in individuals on drug therapy (eg, for diabetes, hypertension, or dyslipidemia) and/or already in secondary prevention.

Neither duration nor quantification of risk factors taken into consideration.

Because family history of early-onset cardiovascular disease, chronic stress, overweight, diet, and sedentary lifestyle were disregarded, participants felt the assessment was incomplete and did not consider them on an individual basis.

It is demoralizing for people who go to great efforts of prevention to learn they are at high cardiovascular risk.

It is counterproductive to tell someone with several behavioral risk factors for cardiovascular disease- such as smoking, sedentary

lifestyle, or overweight - that their cardiovascular risk is low.
After a mean follow-up period of 14 months, the salient findings of the VIVOPTIM intervention pilot program were that this innovative, individualized, multiprofessional, ranked management of cardiovascular risk factors exerted statistically significant beneficial effects on systolic blood pressure, weight management, smoking, and daily number of steps. ${ }^{7}$

However, these preliminary findings also brought to light several limitations of the cardiovascular risk evaluation using the Framingham equation and notably, after comparative analysis of the characteristics of the three risk-level groups, based on feedback from both the coaches and the participants (Box 1).

The steering committee of the VIVOPTIM project therefore decided to seek an alternative to the Framingham risk score before extending the pilot program to the whole of France. For the reasons mentioned earlier, the SCORE risk scale was not suited so the decision was made to focus on the following aspects:

- Even though they are non-modifiable, two major risk factors, ie, age, and family history of early-onset cardiovascular disease, unquestionably have a role to play in the assessment of individual cardiovascular risk. There is obviously no specific threshold for age but in order to be able to classify the participants, we decided upon $>50$ years of age for men, and $>60$ years for women. A family history of cardiovascular disease (coronary heart disease and sudden cardiovascular death) was considered as early-onset if it occurred before the age of 55 years in the father, a brother, or a son, and before the age of 65 in the mother, a sister, or a daughter.

- Smoking, dyslipidemia, hypertension, and type 2 diabetes are the four modifiable major cardiovascular risk factors. The tobacco-related risk factor denoted current smokers or those having quit within the past 3 years; the dyslipidemia risk factor included all cases of atherogenic dyslipidemia, ie, hyper-LDLcholesterolemia according to the thresholds laid down by the French Health Authorities (Haute Autorité de Santé), or hypo-HDL-cholesterolemia. Hypertension was defined as blood pressure $>140$ / $90 \mathrm{~mm} \mathrm{Hg}$ on several different occasions, and diabetes mellitus as a glycemia level $>7 \mathrm{mmol} / \mathrm{L}$ or glycated hemoglobin $>6 \%$ or post-prandial glycemia $>11 \mathrm{mmol} / \mathrm{L}$. 
- In the evaluation of cardiovascular risk, we also included seven associated risk factors that had less weighting than any of the major cardiovascular risk factors mentioned: obesity (BMI $>30 \mathrm{~kg} / \mathrm{m}^{2}$ ), a sedentary lifestyle (weekly physical exercise $<2.5$ hours on top of daily activities), social deprivation (mainly economic and/or housing vulnerability), chronic inflammatory disease (such as rheumatoid arthritis, HIV, gastro-intestinal inflammatory disorder), kidney disease (renal failure, ie, glomerular filtration rate $<60 \mathrm{~mL} / \mathrm{min}$ ), chronic stress as assessed by a selfreported questionnaire, and an unhealthy, nonMediterranean-type diet.

- It was ultimately decided that the six major, plus seven associated cardiovascular risk factors (with a 0.5 weighting for the latter) should be used in the assessment of cardiovascular risk and the ranking of participants in one of three cardiovascular risk levels: low risk for cardiovascular disease (zero or one cardiovascular risk factor), moderate risk (two or three cardiovascular risk factors), and high risk (at least four cardiovascular risk factors or already in a context of secondary cardiovascular prevention).

- Participants were also given another equally important piece of information - particularly in operational terms - with details of excess risk that is calculated from major cardiovascular risk factors that are all

Table I Parameters Used in the Updated Version of the Cardiovascular Risk Assessment

\begin{tabular}{|c|c|}
\hline Major Cardiovascular Risk Factors & $\begin{array}{l}\text { Associated Cardiovascular } \\
\text { Risk Factors (with a } 0.5 \\
\text { Weighting) }\end{array}$ \\
\hline \multicolumn{2}{|c|}{ Non modifiable (or difficult to modify) risk factors } \\
\hline $\begin{array}{l}\text {-Age }(>50 \mathrm{M},>60 \mathrm{~F}) \\
\text {-Family history of early-onset cardiovascular } \\
\text { disease }(<55 \mathrm{M},<65 \mathrm{~F})\end{array}$ & $\begin{array}{l}\text {-Kidney disease } \\
\text {-Chronic inflammatory disease } \\
\text {-Social vulnerability }\end{array}$ \\
\hline \multicolumn{2}{|c|}{ Potentially modifiable risk factors } \\
\hline $\begin{array}{l}\text {-Current smoking (or quit for }<3 \text { years) } \\
\text {-Atherogenic dyslipidemia } \\
\text {-Hypertension } \\
\text {-Diabetes mellitus }\end{array}$ & $\begin{array}{l}\text {-Sedentary lifestyle } \\
\text {-Chronic stress } \\
\text {-Obesity } \\
\text {-non-Mediterranean diet }\end{array}$ \\
\hline
\end{tabular}

Notes: Low cardiovascular risk: 0 or I cardiovascular risk factor, moderate cardiovascular risk: 2 or 3 cardiovascular risk factors, high cardiovascular risk: $\geq 4$ cardiovascular risk factors or already in a context of secondary cardiovascular prevention. No excess risk: no modifiable cardiovascular risk factor, noteworthy excess risk: I modifiable cardiovascular risk factor, moderate excess risk: 2 modifiable cardiovascular risk factors, severe excess risk: $\geq 3$ modifiable cardiovascular risk factors. Abbreviations: $M$, male; F, female. modifiable through measures of prevention, ie, smoking, atherogenic dyslipidemia, hypertension, and diabetes, and to which were added (with a 0.5 weighting) obesity, a sedentary lifestyle, chronic stress, and an unhealthy diet. The excess risk was null if the individual had no modifiable cardiovascular risk factors, was noteworthy with one modifiable cardiovascular risk factor, moderate with two, and severe with three or more modifiable cardiovascular risk factors.

In the new version of the cardiovascular risk assessment, in addition to the evaluation of risk based on the six major plus seven associated cardiovascular risk factors, participants will therefore also systematically receive information on excess risk, ie, risk factors that are preventable via measures of prevention (Table 1).

In conclusion, the choice of a cardiovascular risk scale is contingent on the specific objectives. In an individualized program of prevention such as the VIVOPTIM project, although the initial evaluation should be as detailed as possible, it should focus primarily on risk scoring to rank individuals into different categories, thereby triggering targeted action plans. Whether an individual has a $2.5 \%$ or 3.7\% 10-year risk of developing an atherothrombotic cardiovascular event is irrelevant.

The second step, which is particularly important from an operational perspective, is to focus on modifiable risk factors and to provide constructive information and encouragement thus enabling each individual to bring about the necessary lifestyle changes to reduce their own cardiovascular risk. The VIVOPTIM program has been implemented throughout France since July 2018. The coming years will show us how effectively this approach has improved outcomes.

\section{Ethics Approval and Informed Consent}

An academic scientific committee validated the main study design, including the content of the website and the e-coaching. Both the program and the study received approval from the appropriate ethics and administrative committees: the Comité consultatif sur le traitement de l'information en matière de recherche dans le domaine de la santé (CCTIRS; French Advisory Committee on Information Processing in the Field of Health) (Dossier no. 15.216 bis dated 13 May 2015), and the Commission nationale informatique et libertés (CNIL; French Data 
Protection Authority) (Délibération no. 2015273 dated 13 July 2015).

\section{Acknowledgments}

The authors would like to thank everyone involved in the VIVOPTIM program: the VIVOPTIM project management team and the dedicated MGEN Directors, the operational partners, the professional coaches and, of course, all the volunteers who accepted to take part in the VIVOPTIM experience. This program would not have been possible without the commitment of the Corporate Governance of the MGEN Group. Special thanks go to Isabelle Hébert, Chief Executive Officer of the MGEN Group, an inspiring entrepreneur who constantly strives to improve the health care of subscribers. The authors thank Moyra Barbier for editorial assistance.

\section{Funding}

The MGEN supported all expenses of the VIVOPTIM program.

\section{Disclosure}

All authors received honoraria from the MGEN for their participation as members of the VIVOPTIM committees. JB has received research support or has served on advisory boards or as a speaker for Abbott, Amgen, Astellas, Astra-Zeneca, Bayer, Boehringer Ingelheim, Bouchara-Recordati, Daiichi
Sankyo, Ferring, Gilead, Icomed, Medexact, Medtronic, Novartis, Novo Nordisk, Quantum Genomics, Saint Jude, Sanofi Aventis, and Servier, all outside the context of this work. The authors report no other conflicts of interest in this work.

\section{References}

1. Boulat T, Ghosn W, Morgand C, Falissard L, Roussel S, Rey G. Main trends in cause-specific mortality in mainland France between 2000 and 2016. Bull Epidemiol Hebd. 2019;29-30:576-584.

2. Zins M, Goldberg M; CONSTANCES team. The French CONSTANCES population-based cohort: design, inclusion and follow-up. Eur J Epidemiol. 2015;30(12):1317-1328. doi:10.1007/ s10654-015-0096-4

3. Dibie A, Femery V, Dubois A, et al. VIVOPTIM: retour d'expérience d'un programme expérimental de esanté et de prévention primaire du risque cardiovasculaire global chez des sujets volontaires âgés de 30 à 70 ans [VIVOPTIM: feedback of an e-health experimental program of primary prevention of cardiovascular risk on 30 to 70 years old volunteers]. Ann Cardiol Angeiol. 2018;67:293-299.

4. Available from: www.vivoptim.com. Accessed October 18, 2021.

5. D’Agostino RB Sr, Vasan RS, Pencina MJ, et al. General cardiovascular risk profile for use in primary care: the Framingham Heart Study. Circulation. 2008;117(6):743-753. doi:10.1161/ CIRCULATIONAHA.107.699579

6. Conroy RM, Pyorala K, Sans S, Menotti A, De Backer G, Ducimetière P. Estimation of ten-year risk of fatal cardiovascular disease in Europe: the SCORE project. Eur Heart J. 2003;24:987-1003. doi:10.1016/S0195-668X(03)00114-3

7. Blacher J, Femery V, Thorez F, et al. A novel personalized approach to cardiovascular prevention: the VIVOPTIM programme. Arch Cardiovasc Dis. 2020;113:590-596. doi:10.1016/j.acvd.2020.02.005
Vascular Health and Risk Management

\section{Publish your work in this journal}

Vascular Health and Risk Management is an international, peerreviewed journal of therapeutics and risk management, focusing on concise rapid reporting of clinical studies on the processes involved in the maintenance of vascular health; the monitoring, prevention and treatment of vascular disease and its sequelae; and the involvement

\section{Dovepress}

of metabolic disorders, particularly diabetes. This journal is indexed on PubMed Central and MedLine. The manuscript management system is completely online and includes a very quick and fair peerreview system, which is all easy to use. Visit http://www.dovepress. com/testimonials.php to read real quotes from published authors. 